\title{
Selecting a Component with Longer Mean Life Time in Bivariate Pareto Models
}

\author{
Parameshwar V. Pandit, Shubhashree Joshi \\ Department of Statistics, Bangalore University, Bangalore, India \\ Email: panditpv12@gmail.com, shubhashreejoshi13@gmail.com
}

Received 10 June 2015; accepted 20 July 2015; published 23 July 2015

Copyright (C) 2015 by authors and Scientific Research Publishing Inc.

This work is licensed under the Creative Commons Attribution International License (CC BY). http://creativecommons.org/licenses/by/4.0/

(c) (i) Open Access

\begin{abstract}
In any parallel system, selecting a component with longer mean lifetime is of interest to the researchers. Hanagal (1997) [1] discussed selection procedures for a two-component system with bivariate exponential (BVE) models. In this paper, the problem of selecting a better component with reference to its mean life time under bivariate Pareto (BVP) models is considered. Three selection procedures based on sample proportions, sample means and maximum likelihood estimators (MLE) are proposed. The probability of correct selection for the proposed procedures is evaluated through Monte Carlo simulation using normal approximation. The asymptotic relative efficiency (ARE) of the proposed procedures is presented to facilitate the evaluation of the performance of procedures.
\end{abstract}

\section{Keywords}

Asymptotic Relative Efficiency (ARE), Better Component, Bivariate Pareto, Probability of Correct Selection

\section{Introduction}

The problem of determining the component with longer life time in a two-component parallel system when the two components are dependent is of interest in the present context. The component which has longer mean life time is considered to be a better component. Hanagal [1] considered selecting the better component in a parallel system with two dependent components when the joint distribution life time of the components is bivariate exponential (BVE) distribution. Hanagal [1] considered BVE distribution proposed by Freund [2], Marshall-Olkin [3] and Block-Basu [4]. Hyakuntake [5] considered the above problem when $\left(X_{1}, X_{2}\right)$ follows BVE distribution of Marshall-Olkin. However selection of the better component when $\left(X_{1}, X_{2}\right)$ follows other than BVE has not been considered in the literature. 
The main aim of this paper is to select a best component with reference its life length in a two component parallel system developing a proper statistical tool. Here, the components of the system are assumed to be dependent and their lifetimes follow bivariate Pareto distribution.

The problem of selecting the component in a two dependent component parallel system when life times $\left(X_{1}, X_{2}\right)$ of two components follow bivariate Pareto (BVP) distribution is considered in this paper. Three selection procedures are proposed and their probabilities of correct selection are evaluated.

\section{Selection Procedures}

Veenus and Nair [6] proposed BVP model with survival function

$$
\begin{aligned}
\bar{F}\left(x_{1}, x_{2}\right) & =P\left[X_{1}>x_{1}, X_{2}>x_{2}\right] \\
& =\left(\frac{x_{1}}{\beta}\right)^{-\theta_{1}}\left(\frac{x_{2}}{\beta}\right)^{-\theta_{2}}\left(\frac{\operatorname{Max}\left(x_{1}, x_{2}\right)}{\beta}\right)^{-\theta_{3}} \quad x_{1}, x_{2} \geq \beta>0
\end{aligned}
$$

where $\theta_{1}, \theta_{2}, \theta_{3}>0$. In this paper, assume $\beta=1$, we get the survival function of $\left(X_{1}, X_{2}\right)$ given by

$$
\bar{F}\left(x_{1}, x_{2}\right)=x_{1}^{-\theta_{1}} x_{2}^{-\theta_{2}}\left[\operatorname{Max}\left(x_{1}, x_{2}\right)\right]^{-\theta_{3}}, \quad x_{1}, x_{2} \geq 1 .
$$

The pdf of $\left(X_{1}, X_{2}\right)$ is given by

$$
f\left(x_{1}, x_{2}\right)= \begin{cases}\theta_{1}\left(\theta_{2}+\theta_{3}\right) x_{1}^{-\left(\theta_{1}+1\right)} x_{2}^{-\left(\theta_{2}+\theta_{3}+1\right)} & x_{1}>x_{2} \\ \theta_{2}\left(\theta_{1}+\theta_{3}\right) x_{2}^{-\left(\theta_{2}+1\right)} x_{1}^{-\left(\theta_{1}+\theta_{3}+1\right)} & x_{1}<x_{2} \\ \theta_{3} x^{-(\theta+1)} & x_{1}=x_{2}\end{cases}
$$

where $\theta=\theta_{1}+\theta_{2}+\theta_{3}$

The above BVP model is not absolutely continuous with respect to Lebesgue measure on $\Re^{2}$ and has a positive probability on the diagonal i.e., $P\left[x_{1}=x_{2}\right]=\frac{\theta_{3}}{\theta}$. The random variables $X_{1}$ and $X_{2}$ are independent iff $\theta_{3}=0$ and $X_{1}$ and $X_{2}$ have identical marginal's iff $\theta_{1}=\theta_{2}$.

Let $\left(X_{1 i}, X_{2 i}\right), i=1,2, \cdots, n$ be a random sample of size $n$ from BVP and let $n_{1}\left(n_{2}\right)$ be the number of observations with $X_{1}<X_{2}\left(X_{1}>X_{2}\right)$ in the sample of size $n$. The distribution of $\left(n_{1}, n_{2}\right)$ is trinomial $\left(n ; \frac{\theta_{1}}{\theta}, \frac{\theta_{2}}{\theta}\right)$ where $\theta=\theta_{1}+\theta_{2}+\theta_{3}$.

We propose three selection procedures:

The first selection procedure $R_{1}$ is based on counts

$R_{1}$ : Select $C_{1}$ as better component if $n_{2}>n_{1}$ and select $C_{2}$ when $n_{2}<n_{1}$.

The second selection procedure is based on the sample means of two lifetimes of the components

$R_{2}$ : Select $C_{1}$ as better component if $\bar{X}_{1}>\bar{X}_{2}$ and select $C_{2}$ as better component when $\bar{X}_{1}<\bar{X}_{2}$

where $\bar{X}_{1}$ and $\bar{X}_{2}$ are the sample means of the lifetimes of the two components $C_{1}$ and $C_{2}$ respectively.

The third selection procedure $R_{3}$ is based on MLE's

$R_{3}$ : Select $C_{1}$ as better component if $\hat{\theta}_{1}<\hat{\theta}_{2}$ and select $C_{2}$ when $\hat{\theta}_{1}>\hat{\theta}_{2}$, where $\hat{\theta}_{1}$ and $\hat{\theta}_{2}$ are the MLE's of $\theta_{1}$ and $\theta_{2}$ respectively. There are no closed form expressions for MLE's and so Hanagal [7] obtained MLE's by either Newton-Raphson procedure or Fisher's method of scoring. The derivation of MLEs for the parameters is given in Appendix.

By the assumption $\theta_{1}<\theta_{2}$ (selecting the component $C_{1}$ ) the probability of correct selection based on three procedures are

$$
\begin{aligned}
& P\left(C S \mid R_{1}\right)=P\left[\frac{n_{2}-n_{1}}{n}>0\right] \\
& P\left(C S \mid R_{2}\right)=P\left[\bar{X}_{1}-\bar{X}_{2}>0\right] \\
& P\left(C S \mid R_{3}\right)=P\left[\hat{\theta}_{2}-\hat{\theta}_{1}>0\right]
\end{aligned}
$$


The exact distribution of $U_{1}=\frac{n_{2}-n_{1}}{n}, U_{2}=\bar{X}_{1}-\bar{X}_{2}$ and $U_{3}=\hat{\theta}_{2}-\hat{\theta}_{1}$ are difficult to obtain but their asymptotic normal distributions can be obtained. By central limit theorem $Z_{1}=\frac{\sqrt{n}\left(U_{1}-\mu_{1}\right)}{\sigma_{1}}$, $Z_{2}=\frac{\sqrt{n}\left(U_{2}-\mu_{2}\right)}{\sigma_{2}}$ and $Z_{3}=\frac{\sqrt{n}\left(U_{3}-\mu_{3}\right)}{\sigma_{3}}$ have asymptotic standard normal with distribution where $\mu_{1}=\frac{\theta_{2}-\theta_{1}}{\theta}, \mu_{2}=\frac{\theta_{2}-\theta_{1}}{\left(\theta_{1}+\theta_{3}-1\right)\left(\theta_{2}+\theta_{3}-1\right)}, \mu_{3}=\theta_{2}-\theta_{1}$

$$
\begin{gathered}
\sigma_{1}^{2}=\frac{\left(2 \theta_{1} \theta_{2}+\theta_{1} \theta_{3}+\theta_{2} \theta_{3}\right)}{\theta^{2}} \\
\sigma_{2}^{2}=\sigma_{2}^{(1)}+\sigma_{2}^{(2)}-2 \sigma_{2}^{(12)}
\end{gathered}
$$

where $\sigma_{2}^{(1)}=\frac{\theta_{1}+\theta_{3}}{\left(\theta_{1}+\theta_{3}-1\right)^{2}\left(\theta_{1}+\theta_{3}-2\right)}, \quad \sigma_{2}^{(2)}=\frac{\theta_{2}+\theta_{3}}{\left(\theta_{2}+\theta_{3}-1\right)^{2}\left(\theta_{2}+\theta_{3}-2\right)}$,

$\sigma_{2}^{(12)}=\frac{\theta_{1}\left(\theta_{2}+\theta_{3}\right)}{\left(\theta_{2}+\theta_{3}-1\right)(\theta-2)}+\frac{\theta_{2}\left(\theta_{1}+\theta_{3}\right)}{\left(\theta_{1}+\theta_{3}-1\right)(\theta-2)}+\frac{\theta_{3}}{\theta-2}-\frac{\left(\theta_{1}+\theta_{3}\right)\left(\theta_{2}+\theta_{3}\right)}{\left(\theta_{1}+\theta_{3}-1\right)\left(\theta_{2}+\theta_{3}-1\right)}$ and

$\sigma_{3}^{2}=\left(I^{11}+I^{22}-2 I^{12}\right)$

$I^{i j}, i, j=1,2,3$ are $(i, j)^{\text {th }}$ elements of the inverse of Fisher information matrix $I^{-1}\left(\theta_{1}, \theta_{2}, \theta_{3}\right)$ Hence

$P\left(C S \mid R_{i}\right)=P\left(Z_{i}>-c_{i}\right)=1-\Phi\left(-c_{i}\right)=\Phi\left(c_{i}\right), i=1,2,3$ where $\Phi(\cdot)$ is the cumulative distribution function of standard normal distribution, $c_{i}=\frac{\sqrt{n} \mu_{i}}{\sigma_{i}}$. If $c_{i}>c_{j}$, then the selection procedure $R_{i}$ is better than $R_{j}$, $i \neq j=1,2,3$.

\section{Determination of Minimum Sample Size and Asymptotic Relative Efficiency (ARE)}

The probability requirement based on the selection procedure $R_{i}, i=1,2,3$ is $P\left(C S \mid R_{i}\right) \geq P^{*}$ where $\frac{1}{2}<P^{*}<1$ is fixed constant.

That is, $P\left(C S \mid R_{i}\right) \geq P^{*}$ or $\Phi\left(c_{i}\right) \geq P^{*}$ or $m_{i} \geq \frac{\sigma_{i}^{2} Z_{p}^{2}}{\mu_{i}^{2}}, i=1,2,3$; where $Z_{p}$ is the solution of $\Phi\left(c_{i}\right)=P^{*}$.

The minimum sample size required for the selection procedure $R_{i}$ is $m_{i}=\frac{\sigma_{i}^{2} Z_{p}^{2}}{\mu_{i}^{2}}, i=1,2,3$.

The ARE of the selection procedure $R_{i}$ with respect to the selection procedure $R_{j}$ is given by

$$
\operatorname{ARE}\left(R_{i}, R_{j}\right)=\frac{m_{j}}{m_{i}}=\frac{\sigma_{j}^{2} \mu_{i}^{2}}{\sigma_{i}^{2} \mu_{j}^{2}} .
$$

The AREs are presented in Table 1 for some combinations of $\left(\theta_{1}, \theta_{2}, \theta_{3}\right)$

Table 1 gives the efficiency of three procedures $R_{1}, R_{2}$ and $R_{3}$. The efficiency comparison would be useful in choosing an appropriate procedure.

\section{Some Remarks and Conclusions}

1) It is observed from the table that the selection procedure $R_{2}$ based on sample means performs better than the other two selection procedures $R_{1}$ and $R_{3}$. 
Table 1. The AREs for some combinations of $\left(\theta_{1}, \theta_{2}, \theta_{3}\right)$.

\begin{tabular}{cccc}
\hline Parameters & ARE $\left(R_{3}, R_{1}\right)$ & ARE $\left(R_{2}, R_{3}\right)$ & ARE $\left(R_{2}, R_{1}\right)$ \\
\hline$\theta_{3}=1.01$ & & & \\
$\theta_{1}=1.02, \theta_{2}=1.05$ & 1.0950 & 4.6641 & 5.1072 \\
$\theta_{1}=1.03, \theta_{2}=1.02$ & 1.0952 & 5.0025 & 7.4794 \\
$\theta_{1}=1.04, \theta_{2}=1.00$ & 1.0954 & & 7.9455 \\
$\theta_{3}=1.02$ & & & 4.5561 \\
$\theta_{1}=1.02, \theta_{2}=1.05$ & 1.0953 & 4.1597 & 4.8471 \\
$\theta_{1}=1.03, \theta_{2}=1.02$ & 1.0954 & 4.4267 & 5.9464 \\
$\theta_{1}=1.04, \theta_{2}=1.00$ & 1.0956 & 5.4259 & 4.1711 \\
& & & 4.4064 \\
$\theta_{3}=1.03$ & & & 3.8080 \\
$\theta_{1}=1.02, \theta_{2}=1.05$ & 1.0954 & 4.0225 & 5.0521 \\
\hline
\end{tabular}

2) The selection procedures $R_{1}$ and $R_{3}$ are equally efficient.

3) The probability of correct selection under selection procedures is computed when the sample size is large and the result is similar to that obtained through AREs.

4) The problem of selecting the best component in multi components parallel system is under progress for multivariate exponential (MVE) and multivariate Pareto (MVP) distributions.

\section{References}

[1] Hanagal, D.D. (1997) Selection of a Better Component in Bivariate Exponential Models. Journal of the Italian Statistical Association, 5, 453-460.

[2] Freund, J.E. (1961) A Bivariate Extension of the Exponential Distribution. Journal of the American Statistical Association, 56, 971-977. http://dx.doi.org/10.1080/01621459.1961.10482138

[3] Marshall, A.W. and Olkin, I. (1967) A Multivariate Exponential Distribution. Journal of the American Statistical Association, 62, 30-44. http://dx.doi.org/10.1080/01621459.1967.10482885

[4] Block, H.W. and Basu, A.P. (1974) A Continuous Bivariate Exponential Extension. Journal of the American Statistical Association, 69, 1031-1037.

[5] Hyakuntake, H. (1992) Selecting the Better Component of a Bivariate Exponential Distribution. Statistics and Decisions, 10, 153-162.

[6] Veenus, P and Nair, K.R.M. (1994) Characterization of a Bivariate Pareto Distribution. Journal of the Indian Statistical Association, 32, 15-20.

[7] Hanagal, D.D. (1997) Note on Estimation of Reliability under Bivariate Pareto Stress-Strength Model. Statistical Papers, 38, 453-459. http://dx.doi.org/10.1007/BF02926000 


\section{Appendix}

Maximum Likelihood Estimators of the parameters $\left(\theta_{1}, \theta_{2}, \theta_{3}\right)$ of BVP distribution The likelihood of the sample of size $n$ is

$$
L=\left[\theta_{1}\left(\theta_{2}+\theta_{3}\right)\right]^{n_{1}}\left[\theta_{2}\left(\theta_{1}+\theta_{3}\right)\right]^{n_{2}} \theta_{3}^{n-n_{1}-n_{2}} \prod_{i=1}^{n} x_{1 i}^{-\left(\theta_{1}+1\right)} x_{2 i}^{-\left(\theta_{2}+1\right)}\left[\operatorname{Max}\left(x_{1 i}, x_{2 i}\right)\right]^{-\theta_{3}} \prod_{i \in A} x_{1 i}
$$

where $n_{1}$ be the number of observations with $X_{1 i}<X_{2 i}$ in the sample of size $n$ and $A=\left\{i \mid X_{1 i}=X_{2 i}\right\}$.

The $\log$ likelihood of $\left(X_{1 i}, X_{2 i}\right) \quad i=1,2, \cdots, n$ is

$$
\begin{aligned}
\log L= & n_{1} \log \theta_{1}+n_{2} \log \theta_{2}+n_{1} \log \left(\theta_{2}+\theta_{3}\right)+n_{2} \log \left(\theta_{1}+\theta_{3}\right)+\left(n-n_{1}-n_{2}\right) \log \theta_{3}-\left(\theta_{1}+1\right) \sum_{i=1}^{n} \log x_{1 i} \\
& +\sum_{i \in A} \log x_{1 i}-\left(\theta_{2}+1\right) \sum_{i=1}^{n} \log x_{2 i}-\theta_{3} \sum_{i=1}^{n} \log \operatorname{Max}\left(x_{1 i}, x_{2 i}\right) .
\end{aligned}
$$

The likelihood equations are

$$
\begin{aligned}
& \frac{\partial \log L}{\partial \theta_{1}}=0 \Rightarrow \frac{n_{1}}{\theta_{1}}+\frac{n_{2}}{\left(\theta_{2}+\theta_{3}\right)}-\sum_{i=1}^{n} \log x_{1 i}=0 \\
& \frac{\partial \log L}{\partial \theta_{2}}=0 \Rightarrow \frac{n_{2}}{\theta_{2}}+\frac{n_{1}}{\left(\theta_{2}+\theta_{3}\right)}-\sum_{i=1}^{n} \log x_{2 i}=0 \\
& \frac{\partial \log L}{\partial \theta_{1}}=0 \Rightarrow \frac{\left(n-n_{1}-n_{2}\right)}{\theta_{3}}+\frac{n_{1}}{\left(\theta_{2}+\theta_{3}\right)}+\frac{n_{2}}{\left(\theta_{1}+\theta_{3}\right)}-\sum_{i=1}^{n} \log \operatorname{Max}\left(x_{1 i}, x_{2 i}\right)=0 .
\end{aligned}
$$

Maximum Likelihood Estimators are obtained solving above likelihood equations simultaneously. One can generate some consistent estimators say $\left(\theta_{1}^{(0)}, \theta_{2}^{(0)}, \theta_{3}^{(0)}\right)$ for the parameters $\left(\theta_{1}, \theta_{2}, \theta_{3}\right)$ and use $\left(\theta_{1}^{(0)}, \theta_{2}^{(0)}, \theta_{3}^{(0)}\right)$ as initial solution in Newton-Raphson procedure or Fisher's method of scoring to obtain MLE's $\left(\hat{\theta}_{1}, \hat{\theta}_{2}, \hat{\theta}_{3}\right)$.

So we choose some consistent estimators as follows

$$
\theta_{i}^{(0)}=\frac{n_{i}}{\sum_{j=1}^{n} \log \operatorname{Min}\left(x_{i}, y_{i}\right)}, i=1,2,3
$$

where $n_{3}=n-n_{1}-n_{2}$.

Hence it is easy to check that $\theta_{i}^{(0)} \stackrel{P}{\longrightarrow} \theta_{i}, i=1,2,3$. The elements of Fisher information matrix are given by

$$
\begin{aligned}
& I_{12}=0, I_{13}=\frac{\theta_{2}}{\theta\left(\theta_{1}+\theta_{3}\right)^{2}}, I_{23}=\frac{\theta_{1}}{\theta\left(\theta_{2}+\theta_{3}\right)^{2}}, \\
& I_{11}=\frac{1}{\theta \theta_{1}}+I_{13}, I_{22}=\frac{1}{\theta \theta_{2}}+I_{23}, I_{33}=\frac{1}{\theta \theta_{3}}+I_{13}+I_{23} .
\end{aligned}
$$

Thus $\sqrt{n}(\underline{\hat{\theta}}-\underline{\theta})$ has an asymptotic multivariate normal with mean vector zero and variance-covariance matrix $I^{-1}(\underline{\theta})$ where $\underline{\theta}=\left(\theta_{1}, \theta_{2}, \theta_{3}\right)$ and $\underline{\hat{\theta}}=\left(\hat{\theta}_{1}, \hat{\theta}_{2}, \hat{\theta}_{3}\right)$. 